\title{
Research on Electrostatic-filtration Radon Elimination Techniques in Underground Space
}

\author{
Li Yuan ${ }^{1, a}$, Shibin Geng ${ }^{2, b}$, Bowen Luo ${ }^{3, c}$, Jingjing Wu ${ }^{4, d}$, Jing Wang ${ }^{5, e}$ \\ 1,2,3,4,5 PLA University of Science and Technology, China

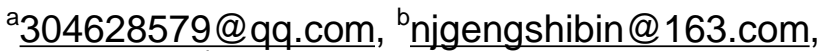

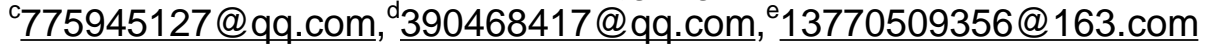

\begin{abstract}
Keywords: radon, radon progeny, static electricity, elimination efficiency
Abstract. Radon and its progeny, lung carcinogens recognized by International Agency for Research on Cancer (IARC) and World Health Organization(WHO), are often obtained to high concentrations in underground space. Based on its decay law, radon and its progeny with bound and unbound state will cause serious damage on health. At present, several common mitigation methods had been taken to eliminate $\mathrm{Rn}$ in the indoor air. However, the environment of underground space often limits the effect of these facilities. Both electrostatic field and filtration are mature technologies in the field of atmosphere purification. Combined with these two different technologies, the electrostatic-filtration radon progeny eliminator is an effective equipment which can eliminate radon and its progeny indirectly. The method is an effective complement to the construction anti-radon technology and ventilation radon elimination technology, and also it is an essential way to solve the problem of radon damage in the underground space.
\end{abstract}

\section{Introduction}

Exposure to radiation of Rn has been confirmed by the U.S. National Academy of Sciences as the second factor [1], with the main factor is smoking, which can contribute to lung cancer. It has also been recognized by international authority such as International Agency for Research on Cancer (IARC), United Nations Scientific Committee on the Effect of Atomic Radiation(UNSCEAR) and World Health Organization(WHO). It has been written in International Commission on Radiological Protection that the damage of radiation root in random takes more than 55\% in one's life and its incubation period endures more than 15 years[2]. According to literatures report, the more exposure time with higher Rn concentration, the more dose of radon could be inhaled. As a result, the possibility to cause cancer will be enhanced with a linear relation[3]. With soil and rock enclosing around, underground space is a suitable place for radon accumulation, especially in the condition of lack of ventilation. Due to different architectonical factor beneath the ground, the radon concentration in the underground space are differences. Cheng[4] make a statistical data show that most of cellars which have been built have their radon concentration exceeding the limitation of $400 \mathrm{~Bq} / \mathrm{m}^{3}$. With the similar architectonical factor researched by Cheng[5], the radon concentration in the underground space is 10 or 100 times higher than over ground part's, which threat subterranean workers' safety seriously. Therefore, it is necessary to create an effective technique to eliminate radon in the underground space.

\section{Common Mitigation Methods and Characters}

The common used mitigation methods including controlling the source of pollution, ventilation and air purification.

\section{Controlling the Source of Pollution}

Most of radon in the underground space result from rock, soil and underground water besides its building envelop[6]. The process of controlling the source of pollution including surveying the building siting, using lower radon-contained building materials and construction sealing. According to the need of surveying the building siting, for one side, there be a limitation of requirement. For the other side, a large number of structures, has been built completely, didn't take radon preventing into concern. The target of using building materials with lower radon-contained 
needs more laws or stipulations to set an indicating level for the construction materials and decorating materials, which can be an essential way to control the safety of newly-built structures[7]. Construction sealing is to setting a whole radon preventing system on the surface of building envelop. It can protect the underground environment with switching off the approaches of outdoor radon-bearing air into the building, blocking the cracks between basement and soil, and also preventing radon-bearing underground water permeating into building[8]. In China, Xiao[9] and colleagues in institution innovated radon preventing paint which have achieved success in some parts. Construction sealing is a permanent method to avoid the source of radon. With the development of sealing materials, the efficiency of building radon preventing is improved successfully. But facing the problem of material aging and the damage during decorating period, this method will be failure to allow radon-bearing air getting through the cracks, which can make an enhance of radon concentration in the building.

\section{Construction Ventilation}

Ventilation is designed to dilute and carry off the indoor radon-bearing air with the supply of fresh air out of the construction. It is the simplest approach to decrease radon concentration in the building effectively[10]. The efficiency of ventilation in the underground space will decline dramatically by utilizing channel ventilation and the zone return system. A study carried out in a building where resort to the channel ventilation and the outdoor air ventilation shows that the radon concentration of the space using the channel ventilation is $343.4 \pm 72 \mathrm{~Bq} / \mathrm{m}^{3}$, while the radon concentration of the space using the outdoor air ventilation is $82.8 \pm 23 \mathrm{~Bq} / \mathrm{m}^{3}$. There is a difference of 4.1 times between them. Furthermore, construction ventilation is limited by seasonal environment factor seriously. Summer is the period that high radon concentration and high humidity show together. There is a contradiction between the methods of radon mitigation and moisture preventing for the reason that if external air is supplied to mitigation radon, removing water from air is necessary to protect underground structure and facilities. As a result, the cost of maintenance and the energy consumption of the building would be increased for the method of ventilation.

\section{Air Purification}

Air purification as a method to eliminate radon has been applied recently. This method, similar with dust removal for air, eliminate dust or particle adsorbed with radon and its progeny, which can achieve the aim of radon elimination indirectly. There are methods to eliminate radon, e.g. fiber filtration technology, adsorption purification and static electricity purification.

Air purifiers sold on markets have been tested by KazukiIwaoka [11] in Japan and it shows that the efficiency of radon mitigation can reach up to $40 \%$ under the supply of aerosol continuously. It is also available to lower the concentration of radon by combine activated carbon filter and high precision fiber filtration together[12]. Nonetheless, high precision fiber increases the resistance of ventilation system while improving radon mitigation efficiency, which will increase the cost of maintenance. By loading electrostatic charge to the fiber, it can increase the effect for eliminate tiny particle and lower the resistance of filtration. However, Boni[13] found that if electrostatic charges vanish gradually, the efficiency of filtration will have a sharp decrease. The disadvantage of activated carbon filter lies on its recycle after saturation, which limited this method be applied to the underground space.

\section{Theory of Electrostatic-filtration Radon Elimination Techniques}

\section{The Character of Radon and Its Progeny}

In ambient temperature, radon can decay to its progeny, a nanoscale metal particle, spontaneously in atmosphere. Radon progeny can deposit on the surface of walls, floors and equipment by gravity, inertia force and Brownian movement. Besides, parts of radon progeny will be adsorbed on the surface of aerosol particle in a short time. These bound state radon progeny might deposit on the surface of each materials and decay continuously at the same time. This period would create more unbound state radon progeny. The existence of both radon and its progeny makes it difficult to 
eliminate them in atmosphere. The key to the problem is to controlling radon progeny especially for unbound state radon progeny. The particle size of unbound state radon progeny ranges from $0.5 \mathrm{~nm}$ to $5 \mathrm{~nm}$, and its average particle size is $1 \mathrm{~nm}$. The particle size of bound state radon progeny ranges from $10 \mathrm{~nm}$ to $1000 \mathrm{~nm}$, and its average particle size is $100 \mathrm{~nm}$. In practical, however, there is a large difference in the particle size of bound state radon progeny due to the source and ingredient of aerosol[14].

Radon gas wouldn't make a serious harm to respiratory system for its long half-life and short period staying in human body. But radon progeny, a metal particle, is inclined to retain in respiratory tract and accumulate in there. Furthermore, radon progeny, the main source of radiological dose, is decaying at the place where it is for its short half-life. Authorizes has acknowledged that radon progeny can cause lung cancer. Therefore, controlling and lowering the concentration of radon in atmosphere make a great effort to reduce the damage of radon progeny to human.

\section{Theory of Radon Elimination Technique}

The theory of electrostatic precipitator can be described below.

Electrostatic precipitator is using the strong static electricity field created by high DC voltage power to ionizing air around and creasing corona. Under the force of airflow, the charged dust suspended in the air is driven to the opposite electrode and in this way reach the aim of separating suspended dust from air. General speaking, the precipitator consists of discharge electrode and collecting electrode. Application of sufficiently high DC voltage would produce a severe uneven electric field that ionize the ambient air of discharge electrode and generate electric corona owing to its highest electric intensity. It means the higher impressed voltage can generate more intense electric corona. A large quantity of free electron and positive ion are generated from the ionization of air in corona zone and due to the decrease of free electron kinetic energy outside the corona zone, it won't make air attached on gas molecules thus forming large amounts of anions caused by collision ionization. When dusty gas enters the electric field, positive ion in the corona zone together with anion outside corona zone collide with dusts and at the same time attach on dusts which realizes the generation of charged dust. Electric field force charged dust to move to the opposite electrode and realize deposition on its surface. As a result, achieve the goal of removing bound state radon progeny indirectly.

The theory of fiber filtration can be described below.

The domain patterns of fiber filtration are sieving, inertia collision, intercepting, diffusion, and also, gravity, electrostatic force and the thermophoresis force. In these factors, electrostatic force is special because aerosol particles often charged with seldom electron. Compared with natural charged particles from friction and decay of radioactive, charged particles from electrostatic force will only get less than $5 \%$ of saturation electricity. As a consequence, less effect can be applied on filtration. But if charging the particle manually, electricity can improve filtration effect obviously. Studies from domain and abroad shows that diffusion and collision play a leading role for the diameter of particles bellow $5 \mu \mathrm{m}$ in the book of Air pollution control engineering[15]. For the particles bigger than $2 \mu \mathrm{m}$, the domain pattern is inertia collision. For those particles which diameter is around $0.5 \mu \mathrm{m}$ to $2 \mu \mathrm{m}$, they situate in the lower limit of inertia collision and intercepting, the upper limit of diffusion. Therefore, it is common that the efficiency for each filtration equipment to collect these kind of particles is extremely low. Combining static electricity technique and fiber filtration can make their effect increasing dramatically to capture the tiny particles, in the range of $0.5 \sim 2 \mu \mathrm{m}$, which is far more effective than any other caption technique. Based on this theory, static electricity is an essential technique for tiny particles caption [16].

In order to cope with radon in both bound and unbound state, actions should be taken. Firstly, deposition of radon progeny bound with aerosol need to be increase. Secondly, unbound state radon progeny in nanometers need to be eliminated as far as possible. Static electricity technique can ionize gas, charge particles and make them transfer. Thus, Static electricity technique has the advantages of depositing and eliminating bound state radon effectively, lower resistance, convent manipulate process and it also can eliminate unbound state radon progeny. At the same time, fiber filtration is installed at the back of electrostatic precipitator in order to collect small-sized unbound 
state radon progeny, which is based on the theory that chemical fiber is inclined to create static electricity during the rubbing of air. This combining can solve the problem of eliminating unbound state radon progeny.

\section{Analysis of the Factors to the Radon Elimination Efficiency}

Electrostatic precipitator can be divided into plate and pipeline according to its shape. Its total caption efficiency can be calculated from the classical Deutsch equation in Theory and technology of dust removal [17]:

$$
\eta_{1}=1-\exp \left(-\frac{L \omega_{e}}{b v}\right)=1-\exp \left(-\frac{A}{Q} \omega_{e}\right)
$$

Where $\mathrm{L}$ is the length of collecting electrode $(\mathrm{m}), \omega_{e}$ is the effective particle migration velocity (ms ${ }^{-}$

$\left.{ }^{1}\right), \mathrm{b}$ is the distance between discharging electrode and collecting electrode plate $(\mathrm{m}), v$ is the air velocity between deposition area, $A$ is the total area of collecting electrode $\left(\mathrm{m}^{2}\right), Q$ is the dealing gas flow $\left(\mathrm{m}^{3} \mathrm{~s}^{-1}\right)$

With Eq. 1 definition, the factors which can affect purification efficiency directly are the total area of collection electrode $A$, the dealing gas flow $Q$ and the effective particle moving velocity $\omega_{e}$. In the common case, once the structure of electrostatic precipitator was designed, the total area of collection electrode $A$ and the dealing gas flow $Q$ will become a constant value. Thus the factor can affect purification efficiency is the effective particle moving velocity $\omega_{e}$ which value is based on many elements such as the particle category, the distribution of particle diameter, air flow velocity, operating voltage, the structure and configuration of electrode and character of power supply. As a result, apart from total quantity of particles, the homogeneity of the particles can also make effects on elimination efficiency.

Fiber material can carry on inner filtration at first, where particles transfer into fiber material. With the possess of filtration running, the particles deposited in fiber material can acting as a sphere capturer, take part in particle collection with fiber afterwards. This phenomenon can be described as dust adsorb dust. As time passed, the quantity of deposited dust increase continually. The efficiency will also change with time and unsteady state process will occur in the inner of fiber. Design Guide for bag filter (2012) gives an efficiency of dust elimination expressing as:

$$
\eta_{2}=1-\exp \left[\frac{4(\varepsilon-1) \delta}{\pi d_{D} \varepsilon} \eta_{\Sigma}\right]
$$

Where $\eta_{2}$ is the efficiency of fiber filtration, $\varepsilon$ is the interspace rate of fiber layer, $\delta$ is the thickness of fiber layer, $d_{D}$ is the diameter of a single fiber, $\eta_{\Sigma}$ is the comprehensive capture efficiency of a single fiber.

Eq. 2 shows that the filtration efficiency of fiber layer is related to the character of fiber and particles, the comprehensive capture efficiency of a single fiber. The comprehensive capture efficiency of a single fiber is affected by inertia efficiency, intercepting efficiency, diffusion efficiency and static electricity efficiency.

Tiny dust can stay longer time in pass through a looser dust layer, which can enhance all kinds of capture efficiency of fiber filtration, especially for diffusion efficiency. That is the leading effect of capturing tiny particles. Consequently, charging particles increase the possibility to capturing particles. On the other hands, condensation of tiny dust resulting from static field makes the diameter of dust particles bigger, which help stopping other particles passing through the fiber material. Static field, meanwhile, accelerate the deposition rate of unbound state radon progeny, which increase the efficiency of capturing unbound state radon progeny.

\section{Electrostatic-filtration Radon Elimination Technology Project}

\section{Project Designing}


Electrostatic-filtration radon progeny eliminator (ESFRPE) is assembled by pre-filtration part, pipeline-electrode field, parallel plate field and fiber filtration part basically. It is designed as a channel structure in order to connect with air-condition system. Each function part can be dismantled respectively for cleaning conveniently. Air go into the machine need to go through a piece of plastic pre-filtration fiber net to have a pre-purification eliminating the bigger particles. Honeycombed and plate field locate in the middle of the machine, where the honeycombed field in ahead and parallel plate field in behind. Air passed through pre-filtration will charge in the honeycombed field firstly and go into parallel plate field secondly, where charged particle be adsorbed by parallel plate field. At last, an electrostatic chemical fiber is set at the back of the ESFRPE. The whole structure is shown in Fig.1

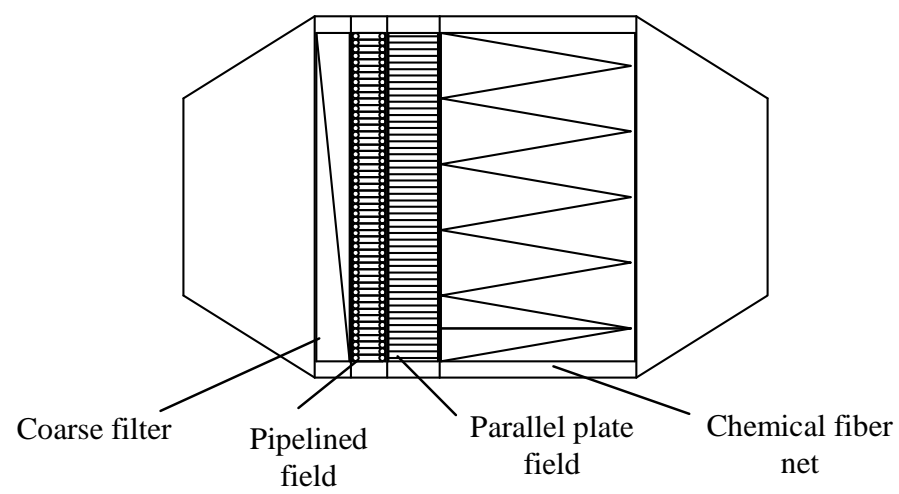

Fig.1 Structure of electrostatic-filtration radon progeny eliminator

\section{Designing of Each Function Part}

\section{Pre-filtration Part}

This part adopts coarse filter in order to eliminate bigger dust and insets in the atmosphere.

\section{Double Electrostatic Field}

In order to offset the disadvantage of pipeline field which is difficult to clean dust, parallel plate field is installed to collecting dust behind the honeycombed field. Thus double field has been built to charging and collecting respectively by pipelined field and parallel plate field. The pipelined field is corona part to charging particle. The following parallel plate field collection part to collecting particle. Owing to the separation of charging and collection field, voltage of the charging part can be adjusted flexible. Through minimize the distance of each electrode, particle can be charged in a lower voltage, which is more security and it is convenient to clean the equipment. Besides, not only is double field effectively, but also it can prevent electrode against anti-corona caused from particle deposition.

One of the significant technology of electrostatic-filtration radon progeny elimination device is to controlling ozone. There are two matter can be applied to control ozone. In one side, choosing lower releasing rate positive electrode, which means apply high voltage in positive electrode and connect the collection electrode with ground. In the other side, choosing strong field and uniformed alternated pipelined field as particle charging area to decreasing ozone releasing. The collection electrode of pipelined electrostatic device in pipeline cylinder, hexagonal prisms or quadrangular. Ionizing needle located in the middle of the pipeline. Among them, honeycombed pipelined field take a cylinder-needle structure, which is convenient to ensuring the distance of needle electrode and cylinder wall are approximately equal in technology, avoiding irregular discharging and control ozone releasing rate as a result.

\section{Fiber Filtration Part}

Sacking form filtration is adapted in this chemical fiber part in order to increase the total surface area of the fiber layer, which can increase the contact area for fiber layer and air flow. Due to 
electrostatic fiber and metal character of radon progeny, Nano-sized unbound state radon progeny can be captured and increase the efficiency of filtrate radon progeny.

\section{Conclusion}

The concentration of radon and its progeny in underground space is far higher than constructions on the ground, which bring a potential risk to workers in the underground space. As a result of the character of high adsorbing ability and short half-life, radon progeny has a vital impact on human's health. Methods, e.g. Source controlling, construction sealing and purifying techniques, have been taken to mitigating radon in the underground space. Double electrostatic field fiber filtration technique can combine these two method's advantages together, optimize the design of radon elimination device for the characters in the underground space, which can enhance the efficiency of radon elimination effect indirectly.

\section{References}

1. Naomi HH, Edith SR. Radon and leukemia in the Danish study: another source of dose[J]. Health Phys, 2009, 97:343-347.

2. International Commission on Radiological Protection, Recommendations of the ICRP, vol. 103, ICRP Publication, Elsevier, 2008.

3. Antony R. Denman, Stephen Rogers, Akeem Ali, John Sinclair, Paul S. Phillips, Robin G.M. Crockett, Christopher J. Groves-Kirkby. Small area mapping of domestic radon, smoking prevalence and lung cancer incidence e A case study in Northamptonshire, UK[J]. Journal of Environmental Radioactivity, 2015, $150: 159-169$

4. CHENG Yexun, WANG Nanping, HOU Shengli, et al. Theoretical Research on Source of Radon from Earth into Air[J]. Radiation Protection Bulletin, 2001, 21(3).

5. CHENG Jing, ZHAN Jianbo, TANG Daikun, et al. Progress of the control for total volatile organic compounds in indoor air pollution [J]. Journal of Public Health and Preventive Medicine, 2011, 24(11):87-92.

6. HUANG Wei, SU Yong. Study on the radon hazard and radon preventing measures in underground building[J]. Shanxi Architecture, 2009, 35(29).

7. Andrey Tsapalov, Konstantin Kovler. Revisiting the concept for evaluation of radon protective properties of building insulation materials[J]. Building and Environment, 2016, 95:182-188.

8. ZHENG Tianliang, YANG Qing, ZHOU Zhuxu, et al. Study on the effect of radon-resistant construction coatings[J].Chinese Journal of Radiological Medicine and Protection, 2005, 25(4).

9. XIAO Detao, ZUO Fuqi, ZHAO Guizhi, et al. Development of an environmental protecting and radon mitigating coating and measurements of its radon mitigating efficiency[J]. Chinese Journal of Radiological Medicine and Protection, 1999, (1)

10. XU Jiaang, LI Fusheng, LU Feng, et al. Study of Reducing the Indoor Radon Dose by Reasonable Control of the Ventilation [J]. Chinese Journal of Radiological Health, 2008, 17(3).

11. KazukiIwaoka, Shinji Tokonami, Tesuo Ishikawa, Hidenori Yonehara. Mitigation effects of radon decay products by air cleaner[J]. J Radioanal NulChem, 2013,295:639-642.

12. Andre Boni, GENG Xinyan. Discussion on the standard and test method of PM2.5 filter[J]. Heating Ventilating \& Air Conditioning, 2013,11:14-16.

13. GUO Hui-ping, SHANG Ai-guo, LU Ning, et al. Introduction to Radon[M].Xi'an: Northwestern Polytechnic University Press,2012:7-14.

14. HE Jiming, MA Guangda, WANG Shuxiao. Air pollution control engineering (Third Edition)[M].Beijing: Higher Education Press, 2010.

15. DAI Ri-jun, LI Wei. Study on mechanism of trapping fine particulate by electrostatic-bag precipitator [J]. Electric Power Environmental Protection, 2011, 27(2): 22-24.

16. XIANG Xiaodong. Theory and technology of dust removal[M]. Beijing: Metallurgical industry press, 2012:132. 
17. YANG Jianxun, ZHANG Dianyin. Design Guide for bag filter[M]. Beijing: China Machine Press, 2012:8. 